\title{
Research on sail control system
}

\author{
Huang Jian ${ }^{1, a, *}$ \\ ${ }^{1}$ XiJing University, Xi'an 710123, China \\ a565200245@qq.com \\ *corresponding author
}

Keywords: Angular displacement sensor; PID algorithm; axial fan

\begin{abstract}
In order to accurately control the wind plate, an experimental platform is built, each about two axial flow fan, PWM wave, wind control plate rotating around a fixed axis, with a high precision angular displacement sensor to measure the rotation angle of 0.1 degrees, high precision. The closed loop system is used to control the duty ratio of PWM wave of fan by PID control algorithm, and the precise control of sail is realized.
\end{abstract}

\section{Introduction}

The wind board control device belongs to the 2015 National Undergraduate Electronic Design Contest control class title, subject requirements to the rotation angle of prefabricated wind plate (between 45 and 135 degrees), 10 seconds to control wind plate reaches a predetermined angle, the deviation is not greater than 5 degrees, the stable time of 5 seconds. In view of the above requirements, double PID control of wind panels has been completed, and the test results have met the requirements.

\section{System Design}

The system design is shown in figure 1. In the picture, the master adopts high-performance microprocessor STM32F103ZET6, which is rich in resources based on Cortex-M3 technology. There are multiple timers, with timer 3 to generate 4 PWM wave, drive 4 BTN7971, control 2 axial flow fan. The angle sensor has a linearity of up to $0.1 \%$ and can accurately measure the angle of rotation. The display unit with 1.44 inch TFT true color screen, using the hardware SPI interface is connected with STM32, it can realize fast refresh ${ }^{[1-3]}$. The power supply unit adopts $3.3 \mathrm{~V}$ direct current.

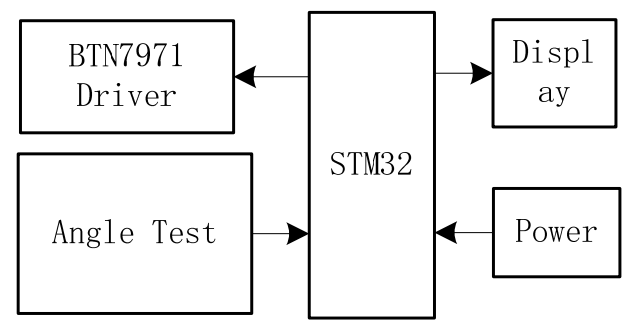

Figure 1 system diagram

\section{Main Hardware Circuit Design}

\subsection{BTN7971 Motor Drive Circuit Design}

BTN7971The motor drive circuit is shown in figure 2. BTN7971 is a Infineon Corporation half bridge motor driver chip. Suitable for driving all kinds of small motor, driving ability is particularly strong, suitable for high current drive. Voltage range from $7 \mathrm{~V}$ to $45 \mathrm{~V}$, the current up to $68 \mathrm{~A}$. The logic control level is 3 to $5 \mathrm{~V}$, which is suitable for connecting various microprocessors. The PC6 and PC7 in the figure are the output pins of the timer 3 of the STM32, which are used as the input 
logic level of the BTN7971 to control the positive, reverse and speed of the motor. The MOTO- and MOTO+ are used as output pins to connect an axial fan to control the speed and direction of an axial fan ${ }^{[4-6]}$. The drive circuit of the other axial fan is the same as shown in Figure 2, and the input logic control is the PC8 and PC9 pins of the STM32.

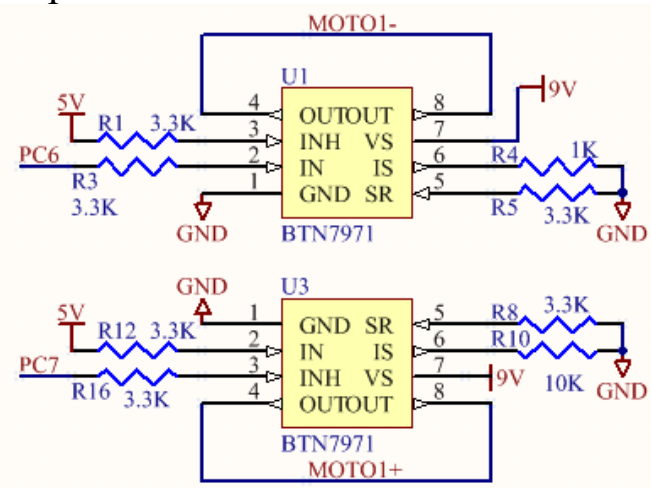

Figure 2 BTN7971 motor drive circuit

\subsection{Design of Rotation Angle Measuring Circuit}

The rotation angle measuring circuit is shown in Figure 3. The rotation angle measurement is implemented with a high precision potentiometer with a resistance value of $5 \mathrm{~K}$ ohms and 2 feet as the output pin. When rotating around the fixed axis, the resistance of the 2 foot will change, [7-8]. The linear error of the resistance value is $0.1 \%$.. When measuring, the $3.3 \mathrm{~V}$ voltage between the 1 feet and the 3 feet is changed, and the resistance value of the 2 foot changes is converted to the corresponding voltage value output, and is input to the A/D acquisition pin PA0 of the STM32 for processing.

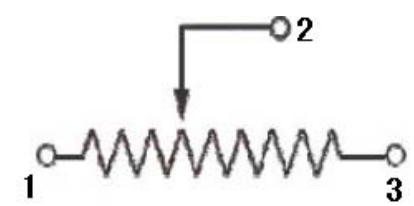

Fig. 3 angle measuring principle diagram

\section{Software Program}

In this design, under KEIL 5, programming with $\mathrm{C}$ language. Completed the measurement of rotation angle, PID control of sail and so on. Some code is given below:

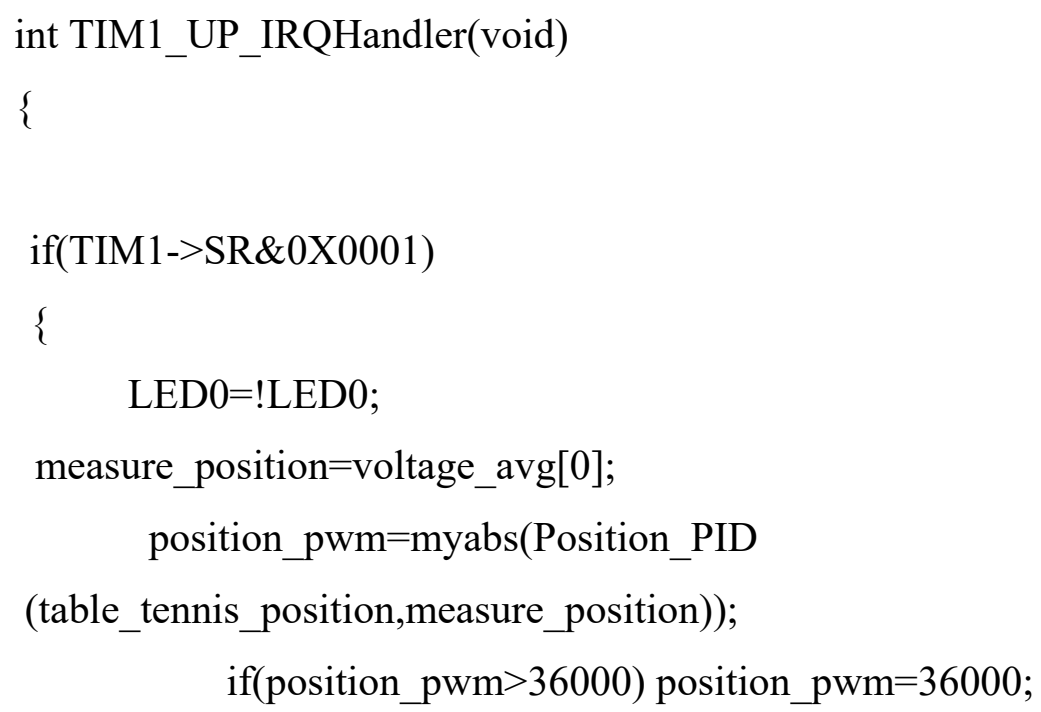


TIM3_PWM_Set(36000,0,3,3,position_pwm);

if(measure_position<table_tennis_position)

\{

position_pwm1=myabs(Position_PID (table_tennis_position,measure_position));

TIM3_PWM_Set(36000,0,3,1,position_pwm1);//to control 90--180angle

\}

else

TIM3_PWM_Set $(36000,0,3,1,0)$;

if $($ position_pwm1 $>36000)$ position_pwm1 $=36000$;

TIM1->SR\&= $(1<<0)$;

\}

return 0 ;

\}

int Position_PID (int Encoder,int Target)

\{

Position_Least $=$ Encoder-Target;

Position_Bias $*=0.8$;

Position_Bias $+=$ Position_Least*0.2;

Integral_bias_p $+=$ Position_Bias;

Position_Differential=Position_Bias-Last_Position;

Position_PWM=Position_Bias*Position_KP+(Position_KI/100.0)*Integral_bias_p +

Position_Differential*Position_KD;

Last_Position=Position_Bias;

return Position_PWM;

\}

\section{Summary}

This paper expounds the principle and method of using PID algorithm to control the wind board, and uses angle displacement sensor to measure rotation angle to form a closed loop system. The PWM output of axial fan is controlled by PID algorithm. The parameters of P, I and D are determined by a large number of experiments, and the rotation angle of the wind plate is accurately controlled, which has certain practical value.

\section{References}

[1] Yu Zebin, Liu Zheng Chong, Lei Ruiting, Zhang Shihong, Chen Wanhua,.2m supersonic wind tunnel structure design and research, [J]. Journal of Aeronautics and aviation, 2013,34 (2): 197-207. 
[2] Jiabing Liu, Li Zhihong, Ren Yanfeng. Modeling and Simulation of Brushless DC motor fuzzy [J]. control engineering of PI system, 2008,15 (S1): 125-133.

[3] Ding Suming, et al. Design and test of.NJS-1 type plant protection DC closed wind tunnel [J]. Chinese Journal of agricultural engineering, 2015,31 (4): 76-86.

[4] Lu Fang Cheng. The natural wind conditions insulator contamination characteristics of [J]. high voltage technology wind tunnel simulation, 2014:40 (5): 1281-1289.

[5] Tang Zhi, et al. Progress in aerodynamic test of hypersonic wind tunnel [J]. Journal of Aeronautics and electronics, 2015:36 (1): 86-97. 